\title{
Nuevos usos del cemento
}

\author{
Barcos de hormigón
}

Dr. Ing. ALFREdo PAEZ BALACA

La reciente crisis del petróleo, una crisis que parece lejos de terminarse, ha impulsado la búsqueda, bien sea de nuevas fuentes de energía o bien de nuevos yacimientos. Con la excepción de algunos países que gozan de unas reservas privilegiadas, los demás han ido ampliando el campo de sus exploraciones a la plataforma costera, adentrándose cada vez más en el océano con la esperanza de encontrar en el subsuelo de las profundidades marinas el ansiado estrato petrolífero o al menos los característicos bolsones de gas que con frecuencia le acompañan.

Desde un punto de vista técnico, la exploración, primero, y la explotación, después, de estos recursos submarinos, plantea la necesidad de construir unas plataformas de perforación tanto más costosas cuanto mayor es la profundidad del fondo, mayor la profundidad del estrato y más hostiles las condiciones del ambiente: bajas temperaturas, violentos temporales o vientos de fuertes intensidades.

Cuando la distancia a la costa es muy pequeña (pozos del lago Maracaibo), el petróleo extraído se almacena en tanques construidos en tierra firme que se enlazan con las torres mediante tuberías. Según las dimensiones, estos tanques de almacenamiento son de hormigón pretensado o metálicos, en sus modalidades de techo fijo o flotante, rodeados de unos cubetos bajos, a modo de tapia, destinados a contener el líquido que, accidentalmente, pueda derramarse en caso de rotura del depósito.

Pero cuando la distancia a la costa es grande, el oleoducto hasta el terminal en tierra supone una fuerte inversión. En estos casos, parece más económico situar el depósito en el fondo marino y construir sobre él la plataforma necesaria para las instalaciones auxiliares de bombeo y carga en los buques petroleros.

Hasta ahora las instalaciones de este tipo recientemente construidas en pleno Mar del Norte, en el Golfo Pérsico y en el de Méjico, son, todas ellas, unas gigantescas botellas de hormigón pretensado. En algunos casos, el empuje violento de las olas se amortigua mediante una pantalla agujereada (muros Jarlan) que rodea al tanque. 
Dadas las condiciones hostiles del ambiente marino, la construcción de estos depósitos se inicia en seco, dentro de un recinto ataguiado, de tal modo que mediante la inundación del mismo en el momento oportuno pueda remolcarse la base del depósito por flotación a un punto próximo a la costa con calado suficiente para proseguir el hormigonado ascendente de la cuba. Ocasionalmente se recurre a un segundo traslado a aguas más profundas para poder terminar la construcción, remolcándose después la estructura hasta su emplazamiento definitivo donde se fondea mediante la inundación de compartimientos periféricos dispuestos a este efecto.

No podemos entrar en el detalle de estas maniobras ni en las características particulares de estas singulares estructuras. Lo que sí es interesante advertir es que nada se opone a la posibilidad de mantener flotante el depósito, anclándolo al fondo cuando la profundidad sea muy grande. Esta solución, que en algunos casos puede ser más económica que la de fondear la estructura a gran profundidad, presenta la ventaja adicional de que, al cabo del tiempo, puede ser desamarrada y transportada a otro lugar sin mayores complicaciones.

Las dimensiones relativas del depósito, especialmente en lo que a la profundidad se refiere, depende en primer término de la permanencia del tanque o depósito en el lugar de destino. Cuanto más frecuentes son los cambios, menor debe ser la profundidad o calado. El depósito deja de ser un silo para pasar a ser una gabarra.

Frente al auge que en estos últimos años han tenido las instalaciones fijas, pocos han sido los intentos realizados en la ejecución de silos transportables. Como excepción cabe señalar el proyecto alemán de un petrolero autopropulsado de hormigón pretensado, pero antes de seguir adelante con este tema conviene reconsiderar el problema bajo un nuevo aspecto.

Otra fuente de energía consiste en la utilización tanto del gas del petróleo como del gas natural. La idea de transportar estos gases en globos dirigibles fue pronto abandonada, más por dificultades de tipo técnico que por fracasos que tuvieron los antiguos zepelines. El sistema de transportar el gas una vez licuado resulta ser más económico, a pesar de los gastos que supone la instalación de unas plantas de licuación más las contrarias de regasificación. Un metro cúbico de líquido equivale a 600 metros cúbicos de gas. En estas condiciones, tanto el transporte como el almacenamiento resultan rentables, a pesar del coste de las citadas instalaciones terminales. El problema técnico que plantea esta modalidad es el referente al almacenamiento de un líquido de bajo peso específico pero a una temperatura de $-163^{\circ} \mathrm{C}$.

A estas temperaturas, tan extremadamente bajas, el acero se torna quebradizo, hasta el punto de que no pueden emplearse aceros al carbono y sólo los aceros aleados con un $9 \%$ de níquel. En estas condiciones la soldadura requiere unos cuidados especiales y unos procedimientos adecuados. Por otra parte, el límite de endurancia del metal desciende notablemente reduciendo fuertemente la capacidad resistente de un acero sometido a cargas repetidas o alternadas.

Frente a estos inconvenientes del acero, el hormigón presenta un sensible incremento de resistencia a esas temperaturas. Cierto es que para resistir los esfuerzos de tracción generados por la presión del líquido contenido, el hormigón necesita ser armado o pretensado, pero los elementos metálicos que en su interior se disponen no son planchas o elementos bidimensionales que resultan afectados por la acritud antes mencionada, sino que son elementos lineales que, en el caso de la armadura pasiva, siempre están en compresión, y en el caso de la armadura activa o de pretensado su régimen tensional es solo ligeramente ondulante con débil proporción de la amplitud de la oscilación comparado con el 
nivel medio de la tensión aplicada. El problema de la posible permeabilidad del hormigón frente a los volátiles gases que se desprenden de la superficie del líquido contenido se resuelve disponiéndose interiormente un escudo metálico de acero al níquel y otro exterior de acero al carbono, pero tanto uno como otro no son elementos resistentes sino unos simples forros de impermeabilización con espesores mínimos. Como norma general, si bien expuesta a las excepciones debidas a las condiciones particulares de la contratación, los grandes tanques para gas natural licuado son de hormigón y los pequeños de acero. Tal vez sea ésta la principal razón por la cual los tanques de los barcos metaneros son, hasta la fecha, bien de aluminio o bien de acero inoxidable, circunstancia que explica el alto coste de estos navíos.

Las ventajosas propiedades del hormigón para soportar bajas temperaturas hace que, en el momento actual, se haya pensado en este material como el más idóneo para la construcción de barcos metaneros. Existe incluso un proyecto para la futura construcción de un barco metanero autopropulsado. Un doble casco, solidarizado entre sí por las cuadernas, constituye el depósito interior, el aislante térmico y el casco exterior. La impermeabilidad del depósito se asegura mediante un forro dejado dentro del casco interior. La impermeabilidad del casco exterior se confía al pretensado.

Un análisis de estas soluciones muestra que un casco de hormigón pesa entre tres y cuatro veces el casco de acero de un barco equivalente. Su coste es sólo del 70 al $80 \%$, pero esta aparente economía en favor del hormigón está mermada por el sobrecoste que supone el transporte de ese aumento de peso. En el caso más corriente de barcos que sólo se detienen en el puerto el tiempo necesario para una carga y descarga rápida, el aumento de peso supone un consumo adicional de combustible que, capitalizado, representa un aumento virtual del coste de primera instalación de un $15 \%$, con lo que los anteriores porcentajes se elevan al 85 y al $95 \%$. Frente a este inconveniente del mayor peso en rosca, el casco de hormigón ofrece la importante ventaja de una mayor duración en perfectas condiciones de servicio y de unos menores gastos de pintura y mantenimiento. En consecuencia la solución de casco de hormigón está tanto más indicada cuanto menor es la movilidad del barco, o dicho de otro modo, cuanto más prolongados sean los períodos de parada en puerto por lentitud de la descarga o por otras razones, por ejemplo su posible función como silo. En estos casos, en los que es lógico pensar que la maquinaria propulsora tiene un bajo rendimiento debido a sus largas horas de inactividad, se recurre con frecuencia a la solución de separar la bodega-silo, de la maquinaria propulsora, transformándose el barco en una gabarra arrastrada o empujada por un remolcador que, siendo independiente por naturaleza, puede atender a otras gabarras. En ciertos tipos de transporte, un remolcador o empujador turna su acción sobre tres gabarras: una en origen en carga, otra en descarga en destino, y una tercera en viaje. Tanto en el caso de barcos metaneros como en éste de gabarras-silo, el empleo del hormigón en el casco y en el recipiente brinda un interesante conjunto de nuevas soluciones abiertas al juego imaginativo del proyectista que busca, en cada material, el medio más idóneo para resolver el problema que tiene planteado.

Es evidente que la función de la estructura o razón por la cual ésta se construye, influye poderosamente en la forma o disposición adecuada a esa finalidad. La inversa también es cierta; la forma de una estructura debe definir, por sí sola, cuál es su tunción. Si al observar la arquitectura de una solución no descubrimos la razón de sus formas, deduciremos que o nuestra sensibilidad está atrofiada o la estructura no es la apropiada tanto en su aspecto funcional como en el artístico.

Esta afirmación no quiere decir que exista una relación biunívoca entre la forma y la finalidad. También el material influye en la forma. Si estos vínculos no están claramente 
expresados, si la estructura no indica su función ni comprendemos la razón del empleo de un cierto material, sentiremos la intranquilidad que nos produce el enigma de un efecto cuya causa ignoramos, o la razón de un mensaje que no comprendemos. Si para facilitar la exposición de unos conceptos, hemos pasado del tanque flotante al petrolero, y de éste a la gabarra, no quiere decir que sus formas son parecidas, ni tampoco que un petrolero metálico tenga la misma forma que un petrolero de hormigón de igual capacidad. El hecho de que en los balbuceos del hormigón se copian las soluciones metálicas, no indica más que la falta de madurez de una idea nacida en una etapa de transición.

José Capmany en sus documentados artículos publicados en la Revista Cemento y Hormigón el año 1971, describe los pormenores de la construcción de las embarcaciones de "ferrocemento" por emplear una palabra directamente extraída del léxico italiano y muy generalizada por su expresividad, aunque debiéramos haberla traducido por "ferromortero", ya que en realidad lo que con este material se construye no es más que un casco compuesto por uno o dos mallazos de barras finas de acero recubiertos por un mortero de pocos centímetros de espesor.

El casco de estas embarcaciones, generalmente de pequeña eslora y modestas dimensiones, tiene la forma clásica del casco de madera. Lo único que se modifica es el material, que ahora se sustituye por otro más permanente y estable. El hecho de que estas embarcaciones de ferrocemento o ferromortero se hayan construido por lo menos en cuatro de los cinco contienentes preferentemente para navegación del interior, fluvial o en los grandes lagos, indica que no son pocos los casos particulares en los cuales el ferromortero e incluso el hormigón armado constituye un verdadero sucedáneo en puntos en donde escasea la chapa o la mano de obra especializada. Con todo hay que reconocer que estos intentos han suministrado una inapreciable fuente de experiencia respecto al comportamiento de estos cascos a lo largo del tiempo, con su secuela de datos referentes a la permanencia de la armadura, recubrimientos, adherencia de moluscos y de algas, rugosidad de las superficies y efecto de rozamiento durante la navegación, reparación de accidentes por choques, incendios e incluso su excepcional comportamiento frente a las explosiones nucleares en el atolón de Bikini (21 de mayo de 1956) y frente a los bombardeos sufridos por varias gabarras de hormigón pretensado durante nueve años de la guerra del Vietnam (Informe Turner).

Conviene advertir que, pese a toda la información hasta la fecha disponible, el intento que ahora hacemos intuir, las soluciones apropiadas partiendo de unas bases distintas como son los depósitos flotantes de hormigón pretensado, nos lleva a la consideración de que la forma de la estructura depende esencialmente de la finalidad de ese "objeto flotante no identificado". Si el transporte es su función primaria, deberá tener proa y popa, independientemente de que sea autopropulsado o no. Si es un silo, habitualmente anclado en un lugar, la proa podrá adoptar la forma de un bisel más o menos apuntado, así como la popa. Las proporciones calado-manga-eslora dependerán de las profundidades en donde se ancle, así como de las condiciones de la carga y descarga a fin de limitar su escora o su variación de calado con el peso almacenado.

La sección transversal dependerá, en primer lugar, de la misión que tiene que cumplir (granelero, aljibe, silo, barco cementero, petrolero, metanero, plataforma de sondeo, planta de licuación, barco-hospital, etc.). La solución clásica de marco rectangular proporciona una bodega amplia pero tiene el inconveniente de su escasa rigidez torsional. La sección bi-tubular es la más apropiada para reducir los giros diferenciales, pero el acondicionamiento de los espacios libres está más limitado. Una solución mixta es la de rigidizar con elementos diagonales el marco rectangular, solución posible cuando se trata de almacenar líquidos. 
El doble casco, unido por elementos transversales a modo de cuadernas, constituye una magnífica solución para silos o cargueros para el transporte de gas natural licuado a $163^{\circ} \mathrm{C}$ bajo cero. Entre ambos cascos se disponen las capas de aislante, generalmente perlita, que queda así encerrada en compartimientos estancos. En el caso de una violenta colisión, estos compartimientos podrán inundarse sin poner en riesgo la estabilidad del buque. Mecánicamente el doble casco funciona como una doble te a efectos de la flexión transversal del costado del casco. En algunos casos estas unidades de doble pared pueden prefabricarse en trozos que se unen entre sí mediante los esfuerzos desarrollados por el pretensado, con las ventajas inherentes a la calidad obtenida por estos procedimientos aptos para la ejecución de unos paños de menos de $0,10 \mathrm{~m}$ de espesor.

Este mismo sistema de la construcción por bloques que se solidarizan por la acción del pretensado es el que se ha seguido en la ejecución del dique de Génova y en la construcción de los diques flotantes de hormigón. Uniendo una sucesión de estos elementos se forman extensas plataformas flotantes que pueden destinarse a la constitución de pistas de aterrizaje y despegue o bien a la formación de puertos más o menos importantes, en general anclados al fondo por unos amarres que permiten orientarle en la dirección del temporal, con lo cual el rompeolas tiene una altura máxima en un limitado desarrollo del muelle.

Con la excepción de estos casos, singulares por su tamaño, lo más frecuente es la construcción de estas estructuras en un dique más o menos convencional con los medios auxiliares acordes con el tamaño del casco y de la posible repetición de idénticos o similares procesos constructivos en unidades semejantes.

Un caso típico de repetición es el de la construcción en la Costa del Pacífico, de gabarras destinadas al almacenamiento de gas natural licuado en depósitos cilíndricos de aluminio, de eje horizontal, algunas de las cuales, las unidades más grandes, incluyen una planta de licuación. Dado que estas instalaciones están destinadas a cumplir su misión en determinados puntos del Pacífico, su disposición general es paralelepipédica, es decir sin proa ni popa, ya que su transporte será muy infrecuente y sólo en especiales distribuciones de carga y en condiciones meteorológicas particularmente favorables.

Son muchas las regiones del globo en las cuales la construcción es particularmente difícil, bien por sus especiales condiciones climáticas o por la escasez de mano de obra generalmente asociada a dificultades en el suministro de materiales. En estos casos, la solución de construir una plataforma flotante, en una zona adecuada, que albergue una determinada instalación industrial y que una vez terminada totalmente se traslada después mediante remolque hasta el punto del litoral elegido, puede tener las ventajas económicas que se derivan de una producción cuyos plazos de ejecución y costes están muy definidos.

Según el número de unidades que haya que construir y según sus dimensiones y analogías entre unas y otras, estas áreas de construcción tendrán una ubicación más o menos definida. En un principio, bastará con localizar una zona del litoral que sea inundable con el calado suficiente para poder maniobrar la estructura construida. La magnitud de este calado, la previsible repetición de esta maniobra y la capacidad de la maquinaria auxiliar que se precisa disponer, definirán las características de estos astilleros de estructuras flotantes de hormigón. En unos casos se requerirán unos diques secos completos y unos muelles de armamento dotados de poderosos medios de elevación. En otros bastará con la excavación de unos recintos defendidos con una ataguía y con la cota de su plataforma a la profundidad conveniente para alcanzar el calado requerido, sin otros medios auxiliares que los habituales en la construcción de estructuras de hormigón. 
Del mismo modo que la técnica del pretensado permite la sólida unión de unos elementos o módulos prefabricados con otros, también la técnica de los hormigones ligeros juegan un importante papel a la hora de decidir el tipo ideal de estructura.

En este caso particular, el término "hormigón ligero" está vinculado al de hormigón de alta resistencia. No se trata de los hormigones ligeros habitualmente empleados en la construcción de viviendas como barrera térmica y acústica de muy bajo peso específico, pero también de escasa resistencia mecánica. Los hormigones a los cuales hacemos referencia son los que algunas veces se denominan como semipesados, es decir hormigones cuya densidad oscila alrededor de $1,85 \mathrm{~kg} / \mathrm{dm}^{3}$ y que, utilizando como árido grueso arcillas expandidas, alcanzan resistencias del orden de los $50 \mathrm{~N} / \mathrm{mm}^{2}$ en probeta cúbica a los 28 días. Su alta dosificación de cemento, entre los 380 y los $420 \mathrm{~kg} / \mathrm{m}^{3}$, su baja relación agua-cemento, su adecuada granulometría que ahora puede fijarse de un modo muy preciso por la homogeneidad en tamaños del árido industrializado y su enérgica consolidación, deben conducir a la obtención de unos hormigones cerrados y compactos, altamente impermeables como conviene a la misión encomendada, resistentes a la acción agresiva de las aguas marinas y con unas magníficas propiedades de aislamiento térmico. El reducido tamaño del árido ligero, 8 ó $16 \mathrm{~mm}$, simplifica la colocación de las masas vertidas envolviendo con facilidad la densa armadura con recubrimientos de 20 a $25 \mathrm{~mm}$ situadas en el interior de unas paredes de escaso espesor.

La arena para estos hormigones también deberá ser de alta calidad, limpia y resistente. La arena de playa queda proscrita a causa del fuerte contenido de cloruros que impregna su superficie. Sólo en casos especiales podrá utilizarse después de comprobarse que, mediante un enérgico lavado con agua dulce, el porcentaje de sales disueltas es inapreciable.

Con el fin de alcanzarse las severas condiciones de durabilidad que se exigen para estos hormigones en contacto con el mar, el cemento utilizable deberá ser un portland, de calidad 450 o superior, cuyo contenido de aluminato tricálcico sea inferior al $10 \%$ pero superior al $5 \%$. La experiencia indica que estos cementos, con las dosificaciones antes indicadas, proporcionan normalmente un satisfactorio comportamiento a lo largo del tiempo en este tipo de estructuras. Por debajo del límite inferior, la durabilidad del hormigón sigue manteniéndose, pero al reducirse la alcalinidad disminuye la protección que el aluminato tricálcico ejerce sobre las armaduras proporcionándoles la adecuada pasivación anticorrosiva.

Desgraciadamente la técnica de los hormigones ligeros no está en España todo lo desarrollada que fuera de desear. Para ser sinceros deberíamos decir que actualmente estamos en los principios de sus aplicaciones y que son pocas las instalaciones nacionales que fabrican las arcillas expandidas. Fuera de nuestras fronteras su empleo ha sido mucho más dilatado y amplio pudiéndose leer en la bibliografía sobre el tema todo tipo de realizaciones, algunas de ellas tan espectaculares como el hangar para la Lufthansa en Frankfurt. No obstante forzoso es reconocer que, por tratarse de una técnica relativamente moderna, estamos faltos de la experiencia a largo plazo. Concretamente sabemos que los módulos de elasticidad del hormigón son más bajos que de costumbre, y más grandes las deformaciones lentas como cabía suponer. Esta circunstancia hace que las pérdidas de pretensado sean mayores que en los hormigones normales, lo cual implica la necesidad de mantener una prudente actitud a la hora de definir los niveles de pretensado.

En cierto modo esta situación, característica de toda técnica moderna, no debe desanimarnos encerrándonos en una posición de desconfianza y de espera. Si queremos incorporarnos a las corrientes del momento actual no debemos permanecer inactivos sino, al 
contrario, adoptar la actitud dinámica de participación en los estudios que están en marcha, y desarrollar nuestros propios ensayos con la amplitud de miras necesaria para que los resultados que se obtengan no sólo sirvan para refrendar la calidad de nuestros productos sino que añadan algo al quehacer de los demás.

Y para eso no basta con el entusiasmo de unos pocos. Hace falta una generosa colaboración por parte de todos los que nos sentimos comprometidos con esta técnica del hormigón y con la herencia de inquietudes que nos legó aquel hombre extraordinario que se llamó Eduardo Torroja. 


\section{COLOQUIO}

1. Abierto el Coloquio por el Presidente, Sr. ANASAGASTI, lo inició el Sr. CALLEJA, quien se expresó así: supuesta construída una unidad metanera o de licuación, ésta debe mantener durante un cierto tiempo un líquido a unos $160^{\circ} \mathrm{C}$ bajo cero. Pero de vez en cuando, esta unidad tendrá que ser descargada y quedará de vacío durante otro cierto tiempo, con lo que, dados los gradientes de temperatura que se establecen, lógicamente se pueden producir choques térmicos muy fuertes. $\mathrm{Y}$ pregunto - dijo-, primero: estos choques térmicos ¿cómo pueden afectar al pretensado, a la adherencia, a la fluencia, etc.?; y segundo: los movimientos de retracción y expansión térmicas a que dan lugar los gradientes ¿de qué forma se puede manifestar y cómo pueden afectar a la estructura?

El Sr. PAEZ contestó diciendo que la solución estaba en la aplicación de la técnica que se utiliza para los tanques de gas natural licuado y que consisten en la existencia de una segunda capa o forro interior metálico de acero al cromo-níquel que, en vez de tener los espesores que corresponden a los tanques metálicos, constituye un simple forro de impermeabilización.

En el proceso de carga y descarga que señala el Sr. CALLEJA - continuó diciendo el Sr. PAEZ-, se produce un fenómeno (aparte del problema del gradiente térmico), en virtud del cual una parte del líquido se transforma en gas muy tenue que puede pasar a través de la chapa de acero. Por ello, estos forros metálicos interiores de acero al níquel-cromo tienden a ser abandonados, y las nuevas soluciones para la impermeabilización parecen consistir en utilizar plásticos especiales que aislan la capa de hormigón de $30 \mathrm{~cm}$ de espesor, seguida de otra capa fuerte de perlita, de otra de hormigón y, en algunos casos, de un forro exterior de acero al carbono.

Mi duda — continuó el Sr. PAEZ-, es si es verdad eso que dicen los libros de que el coeficiente térmico del hormigón y el del acero son aproximadamente iguales; mi impresión —dijo- es que no, y que depende del árido, que en este caso puede tener mucha importancia, por lo que será preciso utilizar áridos adecuados para que, en efecto, los dos materiales, acero y hormigón, tengan aproximadamente el mismo coeficiente térmico. Hay otro problema - prosiguió-, que no puedo explicar: la razón por la cual a temperaturas próximas a $-40^{\circ} \mathrm{C}$ sorprendentemente el hormigón tiene un coeficiente térmico negativo. Es decir, que al enfriar en un cierto intervalo de temperatura, en lugar de acortarse se alarga.

Pero, aparte de estos problemas — siguió diciendo-, la realidad es que se ha podido llegar sin grandes problemas a soluciones prácticas en la construción de estos depósitos. Respecto de problemas de fluencia, se está en fase de experimentación; y en cuanto a cansancio y fatiga parece que tienen poca importancia, salvo quizás esta última en el caso de los barcos metaneros, en los que por los procesos de carga y descarga puede ser algo mayor.

2. A continuación el Sr. HERNANDEZ GRAU formuló una pregunta acerca del tipo de cemento más idóneo para los barcos de hormigón, y si éste llevaba algún recubrimiento. 
El Sr. PAEZ respondió diciendo que existe una comisión de la Federación Internacional del Pretensado (FIP) llamada "Barcos de Hormigón" y dedicada al tema; que un especialista de ella, noruego de fama internacional, opina que, sobre la base de experimentos realizados en su país, el cemento más adecuado es un portland P-550 cuyo contenido de aluminato tricálcico esté comprendido entre el 6 y el 9 por ciento. La razón de esta limitación - prosiguió el Sr. PAEZ-, es que con bajos contenidos de aluminato tricálcico el hormigón resiste perfectamente al agua de mar, pero no defiende tan bien a las armaduras como cuando se cuenta con la alcalinidad que proporciona el aluminato tricálcico. Dicen - añadió-, que con un cemento portland de alta calidad que cumpla dicha limitación, haciendo un hormigón con relaciones agua/cemento bajas —entre 0,40 y 0,45 extraordinariamente compacto, con dosificaciones de cemento superiores a $400 \mathrm{~kg} / \mathrm{m}^{3}$ - lo cual es posible sin problemas de retracción por tratarse de hormigón que va a estar siempre en ambiente húmedo-, la experiencia acumulada hasta ahora es que los barcos de hormigón resisten mucho más de los 25 años que la industria naval estipula para los barcos metálicos. No se trata - concluyó-, de tener que utilizar cementos especiales, sino cementos portland buenos, de alta calidad.

3. Después intervino el Sr. ARREDONDO en relación con los áridos ligeros diciendo que en 1917, cuando se planteó en los Estados Unidos el problema de hacer barcos de hormigón, se llegó a la conclusión de que el asunto era viable, si se podían conseguir densidades de 1,7 con resistencias de $350 \mathrm{kp} / \mathrm{cm}^{2}$, lo que en aquella fecha no debía ser muy fácil. Y que, puesto que el ponente había hablado de hormigones de $500 \mathrm{kp} / \mathrm{cm}^{2}$, preguntabá: ¿cómo se llega a ellos? ¿qué áridos se emplean?

El Sr. PAEZ respondió que parece ser que, en general, la técnica orienta sobre todo al empleo de áridos ligeros a base de arcillas expandidas, y que en tal sentido es preciso cambiar toda la mentalidad en cuanto a dosificaciones, técnica, etc., al tratarse de un árido artificial, con el que hay que procurar jugar en cuanto a la resistencia del hormigón frente a su peso, e intentar una aproximación a los hormigones de $1.000 \mathrm{kp} / \mathrm{cm}^{2}$, de los que tanto se ha hablado.

El Sr. ARREDONDO dijo que, en efecto, así era; y preguntó si el Sr. PAEZ había visto tales hormigones, a lo que éste respondió que no, pero que esperaba verlos.

4. Acto seguido el Sr. CALLEJA pidió intervenir acerca del tema de los cementos y de los hormigones. En cuanto a los cementos - dijo-, me extraña mucho la respuesta del Sr. PAEZ transcribiendo la del miembro noruego de la FIP - hasta el punto de no entenderla-, en el sentido de que el cemento más idóneo es un P-550 con aluminato tricálcico comprendido entre 6 y 9 por ciento. Entendería mejor -aclaró-, que se optase por un cemento con muy poco o ningún aluminato tricálcico, porque cuanto menos contenga -otras características aparte-, menor es su retracción y mayor es o puede ser la alcalinidad actual y residual de su pasta, ya que cada mol de aluminato tricálcico $\mathrm{C}_{3} \mathrm{~A}$ consume un mol de hidróxido cálcico $\mathrm{CH}$ y 12 moles de agua para hidratarse y formar el aluminato tetracálcico hidratado $\mathrm{C}_{4} \mathrm{AH}_{3}$, según: $\mathrm{C}_{3} \mathrm{~A}+\mathrm{CH}+12 \mathrm{H} \rightarrow \mathrm{C}_{4} \mathrm{AH}_{13}$. Así, pues - prosiguió-, la hidratación del aluminato tricálcico puede tomar cal de la fase líquida de la pasta de cemento; cal que, al ser así fijada, deja de engrosar la reserva alcalina de dicha pasta; y la reserva alcalina - para un $\mathrm{pH}$ dado- es el factor influyente en la protección por pasivación de las armaduras frente a la corrosión. Por otra parte, los constituyentes del cemento que mayor reserva alcalina proporcionan son los silicatos, y de ellos más el tricálcico. Por lo tanto, la mayor protección, correspondiente a un mayor $\mathrm{pH}$ y a una mayor reserva alcalina de cal en la pasta, se conseguiría a igualdad de lo demás, cuanto más bajo fuese el contenido de aluminato tricálcico del cemento y más alta su proporción de silicato tricálcico. Según esto, si la recomendación de la FIP hubiese recaído sobre un cemento P-550 de alto contenido de silicato tricálcico y de bajo o muy bajo contenido de aluminato tricálcico — por ejemplo menor del $5 \%$, como sucede con el 
P-550-Y de acuerdo con la designación del Pliego RC-75 para los cementos portland de la más alta calidad, resistentes al yeso-, lo entendería mejor; y eso, sin entrar en consideraciones de ataque al propio hormigón por el agua de mar, de calor de hidratación, etc. —concluyó el Sr. CALLEJA-.

Después pasó a tratar de los hormigones con resistencia de $1.000 \mathrm{kp} / \mathrm{cm}^{2}$, los cuales —dijo-, se pueden conseguir, si se ha de hacer caso de lo que dicen los japoneses al respecto. $\mathrm{Y}$ ¿cómo? -añadió-. Pues a base de unos áridos escogidos y de unas granulometrías adecuadas y muy cuidadas —que, por otra parte, no son nada del otro mundo-, de unas dosificaciones de cemento superiores a $\operatorname{los} 400 \mathrm{~kg} / \mathrm{m}^{3}$, y de unas relaciones agua/ /cemento muy bajas, del orden de 0,3 e inferiores. $\mathrm{Y}$ ¿cómo conseguir éstas? - prosiguió el Sr. CALLEJA-. Pues mediante un aditivo ad hoc que, en dosis inferiores a un 1 por ciento -aproximadamente de $0,3 \%$ - respecto del peso del cemento, son capaces de convertir las correspondientes pastas puras de cementos portland ordinarios y de altas resistencias, en masas que fluyen como un líquido, viscoso y espeso, pero líquido al fin. En tales condiciones y con tales aditivos "superplastificantes", que en realidad son unos dispersantes reductores de agua que, utilizados a dosis tan bajas dispersan totalmente las partículas de cemento en la pasta del hormigón y dan a éste consistencias muy aceptables, se pueden obtener con relaciones agua/cemento tan bajas como las mencionadas, y con los medios adecuados de compactación, hormigones de alrededor de $1.000 \mathrm{kp} / \mathrm{cm}^{2} \mathrm{de}$ resistencia a 28 días.

5. Después intervino el Sr. CACHAN sobre el aspecto de la evaluación de costos y pesos comparativos entre barcos de hormigón y barcos de acero. Me ha parecido - dijo-, que había una diferencia de costo del orden del $20 \%$, y una relación de pesos de 1 a 3 ó 4. Y preguntó si estas evaluaciones eran genéricas, o se referían a alguna gama más específica de tonelajes o formas geométricas de los cascos; y, asimismo, si en las diferencias puestas de manifiesto por estas evaluaciones estaba contemplado el posible costo del hormigón de tan alta calidad del que se estaba hablando.

El Sr. PAEZ contestó que los datos que él había aportado se referían a un estudio hecho por noruegos y, según ellos y sus estudios, para barcos de igual capacidad los costos de los de hormigón normal vienen a ser del $80 \%$, y los pesos entre 3 y 4 veces los de los barcos de acero.

Además - dijo-, estas diferencias en cuanto a costos serían mayores en España, donde, frente al acero, generalmente sale favorecido el hormigón.

Añadió que en los barcos metaneros el peso del casco es muy secundario, y que lo que importa es la capacidad, ya que se transporta un líquido que pesa poco.

6. Luego intervino el Sr. GARCIA SANMARTIN insistiendo sobre el tema de los "superplastificantes", para decir que el problema de su utilización lo venía estudiando desde hacía dos años, habiendo probado algunos, entre ellos unos de origen alemán que se emplea en dosis del 3 al 6 por ciento del peso del cemento, y al precio de 45 a $50 \mathrm{ptas} / \mathrm{kg}$ -carísimo-. Y que, si bien aumentaban mucho la fluidez de la pasta, no era tanto como para conseguir rebajar la relación agua/cemento hasta valores como los indicados por el Sr. CALLEJA.

En tal sentido preguntó a éste si había productos más avanzados que el que él había venido utilizando.

El Sr. CALLEJA respondió que él conocía principalmente dos: uno de origen alemán — cuyo nombre dió, resultando ser el utilizado por el Sr. GARCIA SANMARTIN-, y otro de origen japonés — cuyo nombre y firma productora escribió en el encerado-. A este último - dijo-, es al que yo me refería al hablar de las dosis y efectos que antes he señalado. 
\%. El Sr. DEL CAMPO dijo después que quería ratificar la bonita idea expuesta por el Sr. PAEZ sobre nuevos usos del cemento, sustituyendo materiales y estructuras metálicas por hormigón, ya que en estos momentos una de las inquietudes mundiales es la construcción de plantas de desalación de agua de mar. Hasta ahora — siguió diciendo-, estas plantas se habían realizado con materiales metálicos, pero a causa de los problemas de corrosión y del agotamiento rapidísimo de las instalaciones, por otro lado muy costosas, se estudia ahora con todo interés la posibilidad de su montaje en hormigón. En este sentido - prosiguió-, nuestro país está colaborando, en un trabajo de ámbito europeo, en el estudio del hormigón para construir plantas de desalación de agua de mar; estudio en el que, aparte de los tremendos problemas de corrosión del hormigón por el agua del mar y por los residuos salinos, concurre el del ataque del hormigón por el agua pura. Esto es una prueba más, creo - concluyó diciendo-, de nuevas posibilidades de usos del cemento en las que los cementeros deben estar intersados y también satisfechos de ellas.

8. En relación con lo acabado de expresar, el Sr. BALAGUER dijo haber visitado en mayo, al Sur de Roma, un laboratorio en el que se realizaban estudios sobre hormigón para plantas desalinizadoras de agua de mar en circuito cerrado. Se ensayaban con buen resultado hormigones de cemento puzolánico hecho con una puzolana especial de contenido muy bajo de alúmina y un clínker de tipo FERRARI, comparable al de algunos de los cementos PAS del PCCH-64 o al de algunos de los P-Y del nuevo Pliego RC-75; y se ensayaban también hormigones de cemento portland normal. Ambos impregnados de polímeros resínicos a base de PVC. Y ambos resistiendo igual, pues se sometían a una temperatura de trabajo de $90^{\circ} \mathrm{C}$; y el inconveniente es que a esa temperatura todos los hormigones impregnados de polímeros se ablandan. Ahora bien -añadió-, de no tener que soportar esa temperatura, el hormigón puzolánico resistiría mucho más que el normal. Y puesto que usted, Sr. PAEZ, ha mencionado que se está pensando en utilizar polímeros para la impermeabilización de los barcos metaneros - dijo el Sr. BALAGUER- ¿puede decirnos si está previsto ensayar el PVC? A lo que el Sr. PAEZ respondió que no tenía información al respecto; y que la que poseía sobre el empleo de polímeros, y a la que había hecho alusión, había que tomarla en el sentido de una posibilidad digna de estudio.

Finalmente, a una aclaración pedida por el Sr. DE LA PEÑA en relación con la acción de los "superplastificantes" en las pastas de inyección de hormigón pretensado, el Sr. CALLEJA respondió que, para hacerse una idea, bastaba considerar que con el superplastificante que había citado, y en la proporción señalada, una pasta de relación agua/ /cemento del orden de 0,25 podía hacerse pasar con la misma fácilidad que otra de relación agua/cemento del orden de 0,40, sin superplastificante.

Y no habiéndose producido más preguntas sobre el tema, el Presidente, Sr. ANASAGASTI, agradeció la actuación del ponente y de los participantes en el Coloquio, que quedó así concluido. 